\title{
Prediction of COVID-19 Social Distancing Adherence (SoDA) on the United States county- level
}

Myles Ingram (1) ${ }^{1 凶}$, Ashley Zahabian ${ }^{2} \&$ Chin Hur $^{1}$

Social distancing policies are currently the best method of mitigating the spread of the COVID-19 pandemic. However, adherence to these policies vary greatly on a county-bycounty level. We used social distancing adherence (SoDA) estimated from mobile phone data and population-based demographics/statistics of 3054 counties in the United States to determine which demographics features correlate to adherence on a countywide level. SoDA scores per day were extracted from mobile phone data and aggregated from March 16, 2020 to April 14, 2020. 45 predictor features were evaluated using univariable regression to determine their level of correlation with SoDA. These 45 features were then used to form a SoDA prediction model. Persons who work from home prior to the COVID-19 pandemic $(\beta=0.259, p<0.00001)$ and owner-occupied housing unit rate $(\beta=-0.322, p<0.00001)$ were the most positively correlated and negatively correlated features to SoDA, respectively. Counties with higher per capita income, older persons, and more suburban areas were positively associated with adherence while counties with higher African American population, high obesity rate, earlier first COVID-19 case/death, and more Republican-leaning residents were negatively correlated with adherence. The base model predicted county SoDA with $90.8 \%$ accuracy. The model using only COVID-19-related features predicted with $64 \%$ accuracy and the model using the top 25 most substantial features predicted with $89 \%$ accuracy. Our results indicate that economic features, health features, and a few other features, such as political affiliation, race, and the time since the first case/death, impact SoDA on a countywide level. These features, combined, can predict adherence with a high level of confidence. Our prediction model could be utilized to inform health policy planning and potential interventions in areas with lower adherence.

\footnotetext{
${ }^{1}$ Department of Medicine, Columbia University Irving Medical Center, New York, USA. ${ }^{2}$ Department of Psychology, Columbia University, New York, USA.

凶email: mai2125@cumc.columbia.edu
} 


\section{Introduction}

n January 2020, a new strain of coronavirus, COVID-19 (SARS-CoV-2) was first reported in the United States. Due to the contagious nature of the virus, a country-wide stay-athome order, issued by the government, was put in place on March 16, 2020. Currently, several versions of the vaccine have been produced by many pharmaceutical companies. However, despite the initial distribution of vaccines, the primary method to fight this virus has been social distancing. More research needs to be done in order to determine whether the vaccines prevent asymptomatic infections, whether vaccinated individuals can spread the disease and how long immunity from the vaccines will last. Until these questions are answered and enough people have been vaccinated to establish herd immunity, health officials recommend social distancing practices to slow the spread of COVID-19 (Mallapaty, 2021; Mayo Clinic Health System, 2021).

Social distancing is when a community abstains from interacting with each other closely so that they can mitigate the transmission of the virus. Social distancing guidelines include staying at least 6 feet away from another person, refraining from gathering in groups, and avoiding visits to non-essential establishments (Centers for Disease Control and Prevention, 2020b). In this study, social distancing adherence (SoDA) is the rate at which a community follows the social distancing guidelines. Being able to prioritize risky populations, defined as populations with lower SoDA scores, can help officials make quicker and more effective decisions on resource distribution and social distancing guidelines and regulations.

Other studies have found that a relationship exists between political and socioeconomic features and SoDA (Allcott et al., 2020; Kavanagh et al., 2020; Painter and Qiu, 2020). Republicanleaning counties were shown to be less adherent to social distancing practices than Democratic counties (Allcott et al., 2020; Painter and Qiu, 2020). Counties with lower per capita income and a high proportion of racial minorities were also found to be associated with lower SoDA (Dasgupta et al., 2020; Kavanagh et al., 2020). All of these studies used mobile phone data to estimate SoDA but did not include the distance between the phones as a measure of human encounters. Other reports have addressed SoDA and the risk of infection through social gatherings using social network-based and probabilistic event-based models, but these studies did not factor in socio-economic features to predict SoDA (Block et al., 2020; Chande et al., 2020).

In the case of additional waves of virus infections and new virus variants, it is important to be able to predict which counties will need more respirators, ventilators, drugs and basic personal protective equipment (PPE), like masks, gloves and gowns. Knowing which locations will need to implement more drastic social distancing regulations is vital to preventing another surge in medical complications and deaths. Lastly, if we know which communities are struggling most to adhere to social distancing, policy makers can make guidelines which make social distancing easier and more accessible for those who need it most.

The aim of our analysis was to develop a prediction tool with improved accuracy to guide future health policy planning. With this prediction tool, policy can be made to mitigate the stress put on the health care infrastructure, control the spread of the virus, and manage economic burden.

\section{Methods}

Model overview. We developed a multivariable bagging regression algorithm to predict the SoDA scores of each United States county using 45 predictor features. This bootstrapping technique was used to improve accuracy on the current data, robustness for unseen data, and decrease overfitting. In each sample of the bagging algorithm, a regression model was trained on an $80 \% /$ $20 \%$ training/test split. Graphs of the counties included in the training set and the test set, respectively, can be found in the supplementary materials (Supplementary Fig. 1a, b). The regression models per sample were then aggregated to form the SoDA model. To rank the degree to which each feature correlates SoDA independently, we used univariable linear regressions between each feature and SoDA and calculated the beta coefficient for every regression. Using this result, we were able to create a subsequent model using the top 25 most substantial features on the model in order to determine if reducing the number of features had an effect on model performance. A model using features only related to the COVID-19 pandemic was also created to determine the effect of these features on SoDA prediction. For this version of the model, we solely used the cumulative COVID19 case and death toll for each county, the days since a state issued Stay-At-Home order if applicable, and the days since the first COVID-19 case and death in each county.

Unacast social distancing data. The data used to estimate the degree to which counties were adhering to social distancing was taken from mobile phone movement and location data provided by Unacast (Unacast, 2020). Unacast transformed their mobile phone data into a grading system for SoDA based on three different metrics. The three metrics that comprise the Unacast social distancing score were percent difference in average distance traveled compared to pre-COVID-19 period (Metric 1), percent difference in visitation to non-essential places of interest (restaurants, retail centers, etc.) compared to pre-COVID-19 period (Metric 2), and the rate of human encounters per square kilometer compared to the national average (Metric 3). Pre-COVID19 was defined as the 4 weeks prior to March 8 (February 9, 2020 to March 8, 2020). Devices were ascribed to counties based on where the device was for the longest period of time on a specific day, which accounts for both the difference between work and home and for the people who moved to other places to live during the pandemic. Each metric was quantified into a score from 5 being the highest SoDA and 1 being the lowest. More detail on the scoring system can be found in the Unacast US SDS Methodology document listed in the "Resources" section.

Metric 1 was measured by averaging the distance travelled across all devices per county. The metric was calculated for every day as a percent difference from the average distance traveled on the same day in the same county pre-COVID-19. The metric was shown to be strongly correlated with confirmed cases. Metric 2 was calculated for every day as a percent difference in visitation of non-essential places of interest from the baseline visitation in the same county on the same day pre-COVID-19. If a device was shown to be in a non-essential place of interest, it was counted as visiting. A complete list of the non-essential places of interest can be found in the Unacast US SDS Methodology document. Metric 3 was calculated as a percentage difference in the number of close encounters between two devices per square kilometer from the national pre-COVID-19 average. A close encounter of devices was considered to be a spatial distance of $50 \mathrm{~m}$ or less and a temporal distance of $60 \mathrm{~min}$ or less. Only land area was considered when normalizing the number of encounters by area. Metric 3 was included to account for population density. The final social distancing score for a county on a particular day postCOVID-19 was calculated by taking the average of the 3 metrics. The counties that were excluded did not have sufficient mobile phone data to be included into the analysis. SoDA scores were derived from averaging the overall social distancing grade per county from March 16, 2020 (first day of the national stray at 


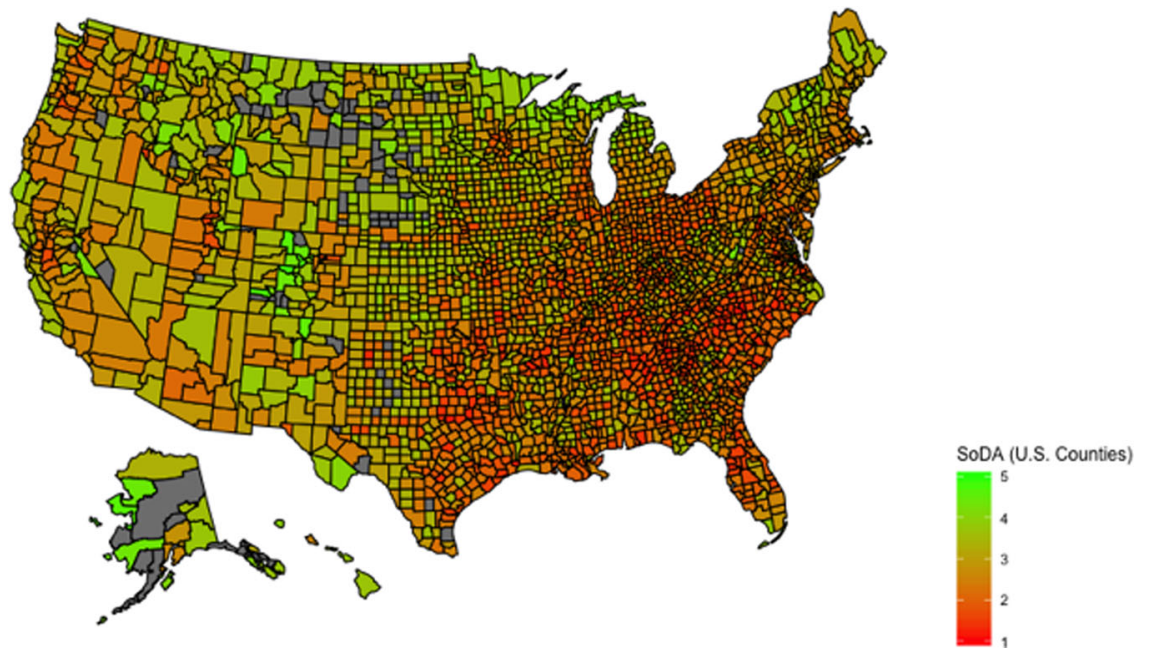

Fig. 1 Social Distancing Adherence (SoDA) heat map by United States county. Authors' analysis of data from the Unacast Social Distancing Dataset between from March 16, 2020 (first day of the national stray at home order) to April 24, 2020 (first day a state relaxed the social distancing guidelines). States that are grey did not have sufficient amount of cellphone data to be included.

home order) to April 24, 2020 (first day a state relaxed the social distancing guidelines). SoDA scores were calculated for 3054 United States counties (Fig. 1).

Socioeconomic data collection. Data on obesity rates, diabetes rates, COVID-19 cases and deaths were collected from the CDC (Centers for Disease Control and Prevention, 2020a). County 2016 presidential election voting data was obtained the MIT Election Data and Science Lab (MIT Election Data and Science Lab, 2018). Data on days since state Stay-At-Home order was scraped from CNN news reports (Rose, 2020). All other predictor features used in the model were collected from American Census Survey (ACS, 5-year averages from 2014 to 2018) (United States Census Bureau, 2020). For all the predictor features, we used the most recent data available as inputs into the model.

Statistical analysis. SoDA scores were expressed as means of daily SoDA scores from March 16, 2020 to April 24, 2020. Univariate regressions analyses were done to assess the association between the features in Table 1 and SoDA scores and obtain the $\beta$ coefficient for the features. The regression analyses were two-tailed with an alpha level of 0.05 . A $p$-value of 0.05 and below was considered statistically significant and the $n$ value of the regressions of the number of counties (3054). Accuracy and goodness of fit of the model were determined by mean squared error and the coefficient of determination, respectively. Algorithms were implemented in Python using scikit-learn library. Analysis code and data repository can be found in the Github link in the "Resources" section.

\section{Results}

Feature correlations. Owner-occupied housing unit rate was the strongest negatively correlated feature $(\beta=-0.322, P<0.00001)$ with SoDA and persons that work from home $(\beta=0.259, p<$ 0.00001 ) was the strongest positively correlated feature (Table 1). Features related to age had significant correlations with SoDA with percentage of the county population 65 and over $(\beta=0.221$, $p<0.00001)$ and median county age $(\beta=0.204, p<0.00001)$ being among the top 25 most substantial features, with both features being positively associated with SoDA scores. Both days since the first COVID-19 case in a county $(\beta=-0.276, p<$ $0.00001)$ and days since the first COVID-19-related death $(\beta=$ $-0.202, p<0.00001)$ were negatively associated with SoDA scores. Several features related to economic status and commuting habits such as persons owning a house $(\beta=0.185$, $p<0.01)$, unemployment rate $(\beta=-0.067, p<0.00001)$, per capita income $(\beta=0.0279, p=0.0279)$, persons owning a vehicle to commute $(\beta=-0.272, p<0.00001)$, and mean travel time to work $(\beta=-0.124, p<0.00001)$ also correlated strongly with county social distancing. Additionally, the number of votes for the Republican presidential candidate in $2016(\beta=-0.137, p<$ $0.00001)$ and percentage of Black/African American populations in a county $(\beta=-0.134, p<0.00001)$ were both significantly negatively correlated with the level a county adhered to social distancing guidelines. Beta coefficients and $p$-values of features used in the top 25 features model are shown in Table 1. The features that did not significantly correlate with SoDA scores were cumulative COVID-19 deaths $(\beta=0.0124, p=0.328)$ and cases ( $\beta=0.00756, p=0.552)$, persons with bachelor's degree or higher $(\beta=-0.00208, p=0.87)$, persons without health insurance $(\beta=$ $-0.0239, p=0.06$ ), and percentage of Chinese/Hispanic populations in a county $(\beta=-0.00292, p=0.818$ and $\beta=-0.00252$, $p=0.842)($ Table 1$)$.

Model results. Using our base SoDA model, COVID-19-related features model, and top 25 feature model, we ran an analysis to predict the SoDA scores of 3054 United States counties from March 16, 2020 to April 24, 2020. The results of these models are shown in heat maps in Figs. 2-4.

Base model. We found that the base SoDA model predicted the SoDA scores of the counties with a $91.6 \%$ accuracy (Fig. 2). The model produced a training accuracy of 91.4\% (Supplementary Fig. 2a), test accuracy of $92.3 \%$ (Supplementary Fig. 2b) and a coefficient of determination of 0.830 on this data.

COVID-19-related features model. The COVID-19-related features model scored an accuracy of $64 \%$ in predicting SoDA scores (Fig. 3). This version of the mode scored an accuracy of $64.1 \%$ on the training set (Supplementary Fig. 3a), an accuracy of $63.9 \%$ on the test set (Supplementary Fig. 3b) and had a coefficient of determination of 0.274 .

Top 25 features model. The model using the top 25 most substantial features, determined by beta coefficient and $p$-value, predicted the county SoDA score with an accuracy of $89.0 \%$ (Fig. 4). The top 25 features model had a training set accuracy of 


\section{Table 1 Social distancing adherence (SoDA) model features.}

\section{Features}

Feature explanation

Correlation coefficient

( $\beta)$

Owner-occupied housing unit rate ${ }^{a}$

Days since first COVID-19 case $^{a}$

Persons using vehicle to commute $(\%)^{\mathrm{a}}$

Persons who work from home (\%)

Households with one or more people under 18 years $(\%)^{a}$

Persons 65 years and over (\%)a

Families with children under 18 years (\%)

Median age (years)

Days since first COVID-19 death ${ }^{\mathrm{a}}$

Persons 65 years and over living alone $\mathrm{e}^{\mathrm{a}}$

Households with one or more people 65 years and over (\%)

Persons owning a home (\%)a

Male population (\%)

Households with Persons Living Alone (\%)

Nonfamily households (\%)

Diabetes rate ${ }^{a}$

Persons 21 years and over $(\%)^{a}$

Votes for Republican presidential candidate

2016a

Black or African American (\%)

Other relatives living in household (\%)

Obesity rate ${ }^{a}$

Mean travel time to work (minutes) ${ }^{a}$

Married families with children under 18 years $(\%)^{a}$

Median gross rent ${ }^{a}$

American Indian and Alaska Native (\%)

Households with a computer (\%)

Average family size

Households with a broadband Internet subscription (\%)

White (\%)

Total housing units

Total population

Persons under 18 with a disability (\%)

Unemployment rate

High school graduate or higher (\%)

Days since state stay at home order

Families in poverty (\%)

Foreign born (\%)

Asian (\%)

Per capita income
Percentage of the housing units in a county in which the owner resides

Number of days that passed since the first COVID-19 case in -0.2758946 the county was identified.

Percentage of the county population that commutes to work -0.2725402 using a vehicle.

Percentage of the county population that works from home prior to pandemic.

Percentage of the county's households with at least one individual under 18 years old.

Percentage of the county population that is 65 years or older. Percentage of the county's families that have a child 18 years old or younger.

Median age in the county

Number of days that passed since the first COVID-19 related death in the county was identified.

Percentage of the county population that is 65 years or older and living alone.

Percentage of the county's households with one or more individual(s) 65 years or older.

Percentage of the county population that owns a home.

Percentage of the county population that is male.

Percentage of the county's households with a person living alone.

Percentage of county's households with none of the inhabitants related

Percentage of county population with diabetes

Percentage of the county population that is 21 years old and over.

Number of votes the Republican presidential candidate

received from the county in 2016

Percentage of the county population that is Black or African American.

Percentage of the county's households with

Percentage of county population that is obese

The mean travel time, in minutes, that it takes an individual living in the county to get to work.

Percentage of the county population's families with children under 18 years old.

The median gross rent of the county.

Percentage of the county population that is American Indian and Alaska Native.

Percentage of the county population's households that have a computer.

The average family size in the county.

Percentage of the county population's households that have a broadband internet subscription.

Percentage of the county population that belongs to the white racial category.

The total number of housing units in the county.

The total population of the county.

0.25866076

0.25866076

$-0.2453643$

0.22056466

$-0.2182014$

0.20389039

$-0.2022794$

0.19635902

0.18856919

0.18475356

0.18305657

0.17324596

0.16739809

$-0.1653597$

0.15300264

$-0.1366638$

$-0.1338838$

$-0.1325252$

$-0.1246405$

$-0.123859$

$-0.120569$

$-0.115392$

0.11320494

$-0.1069481$

$-0.1000168$

$-0.0988313$

0.08425892

$-0.0798798$

$-0.0769589$

Percentage of the county population that is 18 years old or younger with a disability.

Percentage of the county population that is unemployed and part of the labor force.

Percentage of the county population that has graduated high school or higher education.

Number of days that passed since the COVID-19 Stay-AtHome order was put in place in the county.

Percentage of the county population's families that live in poverty.

Percentage of the county population that is born in a foreign country.

Percentage of the county population that is Asian

Mean income for every man, woman, and child
$-0.074821$

$-0.0672901$

0.0631892

$-0.0501806$

$-0.049514$

$-0.0305755$

$-0.0293257$

0.02791053 p-value

$<0.00001$

$<0.00001$

$<0.00001$

$<0.00001$

$<0.00001$

$<0.00001$

$<0.00001$

$<0.00001$

$<0.00001$

$<0.00001$

$<0.00001$

$<0.00001$

$<0.00001$

$<0.00001$

$<0.00001$

$<0.00001$

$<0.00001$

$<0.00001$

$<0.00001$

$<0.00001$

$<0.00001$

$<0.00001$

$<0.00001$

$<0.00001$

$<0.00001$

$<0.00001$

$<0.00001$

$<0.00001$

$<0.00001$

$<0.00001$

$<0.00001$

$<0.00001$

$<0.00001$

$<0.00001$

0.00008

0.00009

0.016

0.021

0.0278 


\section{Table 1 (continued)}

\begin{tabular}{|c|c|c|c|}
\hline Features & Feature explanation & $\begin{array}{l}\text { Correlation coefficient } \\
(\beta)\end{array}$ & $p$-value \\
\hline $\begin{array}{l}\text { Persons with no health insurance } \\
\text { coverage }(\%)\end{array}$ & $\begin{array}{l}\text { Percentage of the county population without health insurance } \\
\text { coverage. }\end{array}$ & -0.0239117 & 0.059 \\
\hline Cumulative COVID-19 deaths & Total number of COVID-19 related deaths in county & 0.012426 & 0.328 \\
\hline Cumulative COVID-19 cases & Total number of COVID-19 cases in county & 0.007555 & 0.552 \\
\hline Chinese (\%) & Percentage of the county population that is Chinese & -0.0029212 & 0.818 \\
\hline Hispanic or Latino (\%) & $\begin{array}{l}\text { Percentage of the county population that is Hispanic } \\
\text { or Latino }\end{array}$ & -0.0025247 & 0.842 \\
\hline Bachelor's degree or higher (\%) & $\begin{array}{l}\text { Percentage of the county population with a bachelor's degree } \\
\text { or higher }\end{array}$ & -0.0020841 & 0.870 \\
\hline
\end{tabular}

Features are ordered in strength of correlation with adherence regardless of positive/negative correlation. A p-value of 0.05 and below was considered statistically significant. Authors' analysis of data from CDC, MIT Election Data and Science Lab, CNN news reports, and American Census Survey (ACS, 5-year averages from 2014 to 2018).

a Features used in the top 25 most substantial features model.

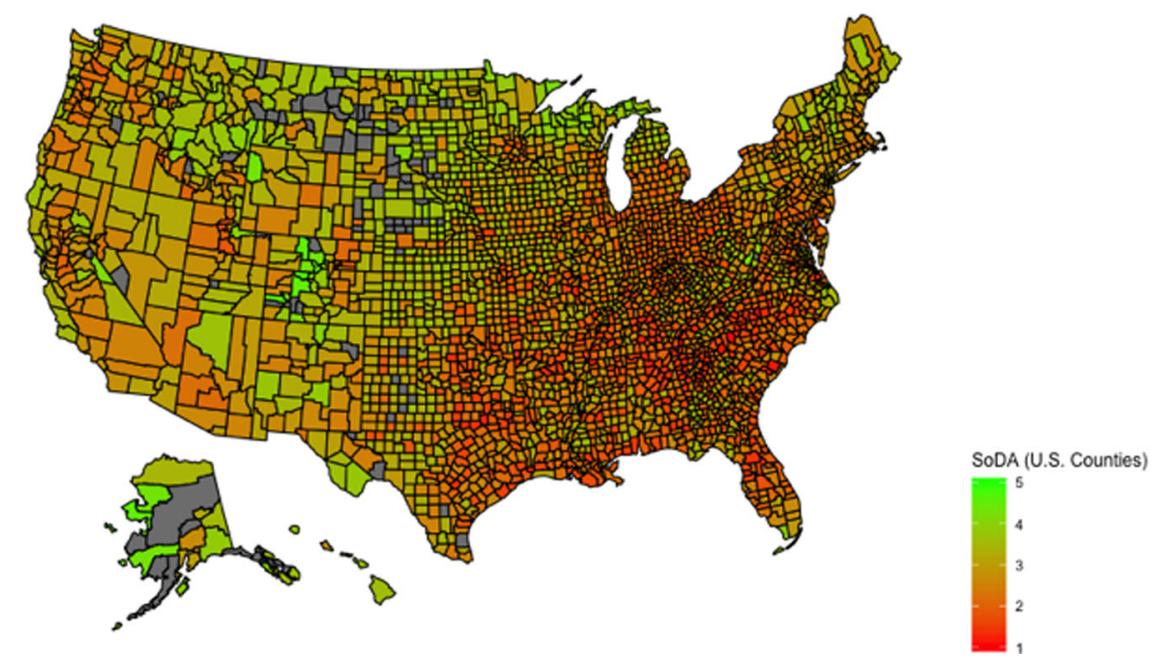

Fig. 2 Base model results heat map by United States county. States that are grey did not have sufficient amount of cellphone data to be included. Authors' analysis of authors' model based on data from the Unacast Social Distancing Dataset between from March 16, 2020 (first day of the national stray at home order) to April 24, 2020 (first day a state relaxed the social distancing guidelines), CDC, MIT Election Data and Science Lab, CNN news reports, and American Census Survey (ACS, 5-year averages from 2014 to 2018).

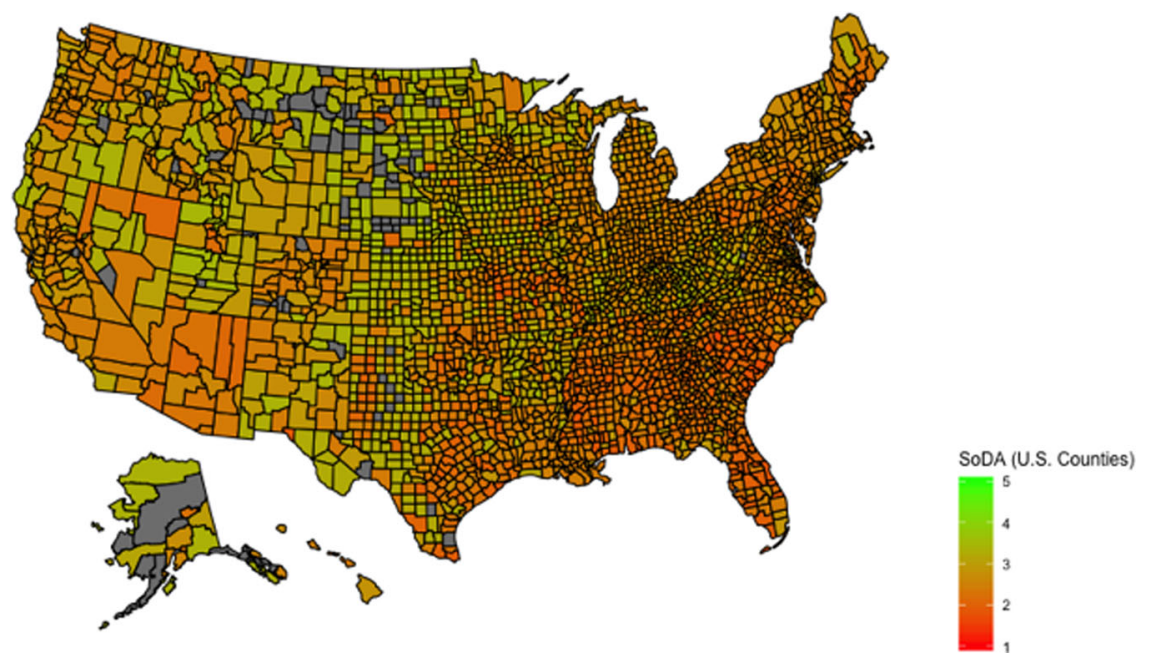

Fig. 3 COVID-19-related features model results heat map by United States county. Authors' analysis of authors' model based on data from the unacast social distancing dataset between from March 16, 2020 (first day of the national stray at home order) to April 24, 2020 (first day a state relaxed the social distancing guidelines), CDC, and CNN news reports. States that are grey did not have sufficient amount of cellphone data to be included. 


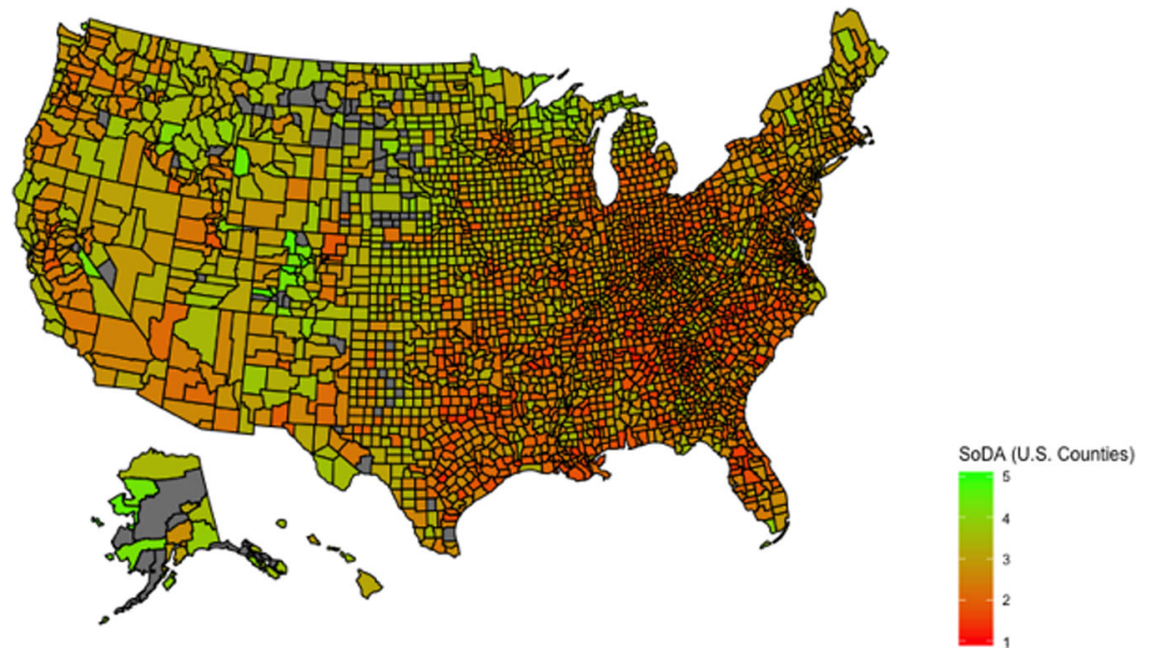

Fig. 4 Top 25 most substantial features model results heat map by United States county. Authors' analysis of authors' model based on data from the unacast social distancing dataset between from March 16, 2020 (first day of the national stray at home order) to April 24, 2020 (first day a state relaxed the social distancing guidelines), CDC, and CNN news reports. States that are grey did not have sufficient amount of cellphone data to be included.

88.7\% (Supplementary Fig. 4a), a coefficient of determination of 0.777 , and a test set accuracy of $89.9 \%$ (Supplementary Fig. $4 \mathrm{~b}$ ).

\section{Discussion}

Our results show that economic features impacted a county's adherence to social distancing. Persons using vehicles to commute and owner-occupied housing rate data indicate that those who live in more suburban areas, who are more likely to use cars to get around or to get to work, have lower adherence. Families in poverty data indicates that those in poverty have a harder time affording the luxury of social distancing, as those with lower incomes feel they need to work in order to survive (Dasgupta et al., 2020). On the contrary, per capita income correlates positively with SoDA, suggesting those with higher incomes are more likely to afford the ability to social distance.

Health features had varied correlations with adherence, particularly among populations who have greater risk of severe illness from COVID-19. Although the diabetic and obese populations are at-risk populations, counties with higher obesity and diabetes rates had lower adherence. Because of the lower adherence, COVID-19related hospitalizations and deaths for people with obesity and diabetes could increase dramatically in these counties. Conversely, we found that, in terms of age, risk of severe illness positively trended with adherence. Communities with persons above 65 years old, who are members of the COVID-19 at-risk population, and a higher median age both had higher adherence. Counties that had a higher rate of individuals aged 18 or younger had lower SoDA, most likely due to the fact that they have stronger immune systems than people above the age of 65 and felt less of a need for social distancing. The cumulative COVID-19 cases and deaths had a negligible impact on predicting SoDA and, furthermore, the model using only COVID-19-related features had lower accuracy than the model with all 45 demographical features. These findings highlight the importance of socioeconomic features in the decision to adhere to social distancing guidelines.

We also found that the proportion of Black/African Americans in a county correlated negatively with SoDA. These findings were consistent with previous studies (Kavanagh et al., 2020; Yancy, 2020). Other studies have shown that nearly half of the COVID-19 cases and 60\% of COVID-19 deaths come from African American (Laurencin and McClinton, 2020; Mahajan and Larkins-Pettigrew, 2020). Furthermore, the percentage of African-Americans living in a county and the percentage of county COVID-19 confirmed were positively correlated (Laurencin and McClinton, 2020; Mahajan and Larkins-Pettigrew, 2020; Yancy, 2020). These findings suggest overcrowding in these communities, which maintaining social distancing guidelines cumbersome and very difficult. Other explanations could be that these communities cannot afford to social distance or the myth of black immunity was detrimental to SoDA.

Other notable features with insightful correlations to adherence included political affiliation for the 2016 presidential election, persons who worked from home prior to the pandemic, and the amount of days that passed since the county's first COVID-19 case and/or death. We found that counties that had more votes for the Republican candidate in 2016 were less likely to follow social distancing guidelines, a result that supports previous findings (Allcott et al., 2020; Kavanagh et al., 2020; Painter and Qiu, 2020). The proportion of the population that worked from home prior to the pandemic had the strongest positive correlation with adherence. A possible explanation for this result is that people who were already working from home had to make less adjustments to their daily routine to adhere to social distancing guidelines compared to people who had to commute to work, thus making social distancing more accessible. We found that the more days that passed since the first COVID-19 case and death, the lower the adherence. A possible explanation for these findings is that the counties with earlier first case/death dates eclipsed the highest case/death rate day earlier, thus diminishing the apparent risk of COVID-19 infection (Qureshi et al., 2020).

In order to best prepare for additional waves and new virus strains, government officials need tools to accurately predict the spread of the virus. Our prediction model using socio-economic data, such as demographic, economic and health data, in addition to COVID-19 death and case tolls can provide accurate predictions of SoDA with a high level of confidence, thus predicting future cases. Additionally, the proposed model can be improved to predict daily social distancing behavior and adherence. In cases where, for example, social distancing occurs less on weekends, states and counties can use this data to formulate and place stricter guidelines on the weekends to avoid setbacks. This prediction model can also be used to strengthen current social network models and/or website risk assessment tools by using more granular socio-economic data (Block et al., 2020; Chande et al., 2020). 
There are some limitations in this model, such that our data does not account for whether someone is wearing a mask or gloves when they are near each other. However, recent studies have shown that wearing a mask, along with handwashing, is associated with adherence to social distancing protocols (Doungngern et al., 2020; Marchiori, 2020). Additionally, the data does not include information about non-phone users. Aggregating the SoDA scores over the course of the quarantine may have caused different results than observing the scores on a daily basis. A future model could explore the features that correlate with daily SoDA. Lastly, the Census data might also not reflect current demographics, as the estimates are as recent as 2018.

In summary, our analysis found that features related to economic status, political affiliation, age, race, diabetes, obesity, and date of first COVID-19 case/death correlated strongly with SoDA of a county. We demonstrated that these demographic features were capable of creating an accurate model for predicting county adherence. This prediction model can be a tool to aid health policy planning in the United States in the case of additional waves of COVID-19 or in the event of another pandemic. Future research is necessary to gain more insight into the underlying reasons for the correlations between the features of the model and SoDA.

Resources. Github Repository: https://github.com/Ingrammyles8/ SoDA_prediction_model

Unacast US SDS Methodology: https:/github.com/Ingrammyles8/ SoDA_prediction_model/blob/master/methodology/US_SDS_ Methodology_Unacast.pdf

\section{Data availability}

All data generated or analyzed during this study are included in this published article and the supplementary files.

Received: 10 September 2020; Accepted: 2 March 2021;

Published online: 23 March 2021

\section{References}

Allcott H, Boxell L, Conway J, Gentzkow M, Thaler M, Yang DY (2020). Polarization and public health: partisan differences in social distancing during the coronavirus pandemic. NCER Working Paper No. w26946.

Block P, Hoffman M, Raabe IJ, Dowd JB, Rahal C, Kashyap R, Mills MC (2020). Social network-based distancing strategies to flatten the COVID-19 curve in a post-lockdown world. Nat Hum Behav 4(6):588-596. https://doi.org/10.1038/ s41562-020-0898-6

Centers for Disease Control and Prevention (2020a). Cases in the U.S. https://www. cdc.gov/coronavirus/2019-ncov/cases-updates/cases-in-us.html. Accessed 12 May 2020.

Centers for Disease Control and Prevention (2020b). Social distancing, quarantine, and isolation. https://www.cdc.gov/coronavirus/2019-ncov/prevent-gettingsick/social-distancing.html. Accessed 12 May 2020.

Chande A, Lee S, Harris M, Nguyen Q, Beckett SJ, Andris C, Weitz JS (2020). Realtime, interactive website for US-county-level COVID-19 event risk assessment. Nat Hum Behav 4(12):1313-1319. https://doi.org/10.1038/s41562-02001000-9.

Dasgupta N, Jonsson Funk M, Lazard A, White BE, Marshall SW (2020). Quantifying the social distancing privilege gap: a longitudinal study of smartphone movement. Public Global Health. Preprint at https://doi.org/10.1101/ 2020.05.03.20084624. Accessed 11 May 2020.

Doung-ngern P, Suphanchaimat R, Panjagampatthana A, Janekrongtham C (2020). Associations between mask-wearing, handwashing, and social distancing practices and risk of COVID-19 infection in public: a case-control study in Thailand. Infect Dis. Preprint at https://doi.org/10.1101/2020.06.11.20128900. Accessed 12 May 2020.

Kavanagh NM, Goel RR, Venkataramani AS (2020). Association of county-level socioeconomic and political characteristics with engagement in social distancing for COVID-19. Health Policy. Preprint at https://doi.org/10.1101/ 2020.04.06.20055632. Accessed 10 Aug 2020.

Laurencin CT, McClinton A (2020) The COVID-19 pandemic: a call to action to identify and address racial and ethnic disparities. J Racial Ethn Health Disparities 7(3):398-402. https://doi.org/10.1007/s40615-020-00756-0.

Mahajan UV, Larkins-Pettigrew M (2020). Racial demographics and COVID-19 confirmed cases and deaths: a correlational analysis of 2886 US counties. J Rac Ethn Health Disparities 7(3):398-402. https://doi.org/10.1007/s40615 020-00756-0.

Mallapaty S (2021). Can COVID vaccines stop transmission? Scientists race to find answers. Nature. https://www.nature.com/articles/d41586-021-00450-z. Accessed 25 Feb 2021.

Marchiori M (2020). COVID-19 and the social distancing paradox: dangers and solutions. Physics. q-Bio ArXiv:2005.12446. http://arxiv.org/abs/2005.12446. Accessed 12 Aug 2020.

Mayo Clinic Health System (2021). Continue to wear a mask, practice social distancing after COVID-19 vaccination. https://www.mayoclinichealthsystem. org/hometown-health/featured-topic/continue-safety-practices-after-covid19-vaccination. Accessed 25 Feb 2021.

MIT Election Data and Science Lab (2018). County Presidential Election Returns 2000-2016. Accessed 30 Apr 2020.

Painter M, Qiu T (2020). Political beliefs affect compliance with COVID-19 social distancing orders. SSRN Electron J. https://doi.org/10.2139/ssrn.3569098

Qureshi AI, Suri MFK, Chu H, Suri HK, Suri AK (2021). Early mandated social distancing is a strong predictor of reduction in peak daily new COVID-19 cases. Public Health. 190:160-167. https://doi.org/10.1016/j.puhe.2020.10.015.

Rose A (2020). This is where all 50 states stand on reopening. https://www.cnn. com/interactive/2020/us/states-reopen-coronavirus-trnd/. Accessed 15 Aug 2020 .

Unacast (2020). Unacast social distancing dataset. https://www.unacast.com/datafor-good. Accessed 30 Apr 2020.

United States Census Bureau (2020). https://data.census.gov/cedsci/. Accessed 10 May 2020.

Yancy CW (2020). COVID-19 and African Americans. JAMA 323(19):189. https:// doi.org/10.1001/jama.2020.6548

\section{Competing interests}

The authors declare no competing interests.

\section{Additional information}

Supplementary information The online version contains supplementary material available at https://doi.org/10.1057/s41599-021-00767-0.

Correspondence and requests for materials should be addressed to M.I.

Reprints and permission information is available at http://www.nature.com/reprints

Publisher's note Springer Nature remains neutral with regard to jurisdictional claims in published maps and institutional affiliations.

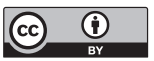

Open Access This article is licensed under a Creative Commons Attribution 4.0 International License, which permits use, sharing, adaptation, distribution and reproduction in any medium or format, as long as you give appropriate credit to the original author(s) and the source, provide a link to the Creative Commons license, and indicate if changes were made. The images or other third party material in this article are included in the article's Creative Commons license, unless indicated otherwise in a credit line to the material. If material is not included in the article's Creative Commons license and your intended use is not permitted by statutory regulation or exceeds the permitted use, you will need to obtain permission directly from the copyright holder. To view a copy of this license, visit http://creativecommons.org/ licenses/by/4.0/.

(c) The Author(s) 2021 